\title{
Wastewater treatment modelling: dealing with uncertainties
}

\author{
E. Belia, Y. Amerlinck, L. Benedetti, B. Johnson, G. Sin, \\ P. A. Vanrolleghem, K. V. Gernaey, S. Gillot, M. B. Neumann, \\ L. Rieger, A. Shaw and K. Villez
}

\section{$\overline{\text { ABSTRACT }}$}

This paper serves as a problem statement of the issues surrounding uncertainty in wastewater treatment modelling. The paper proposes a structure for identifying the sources of uncertainty introduced during each step of an engineering project concerned with model-based design or optimisation of a wastewater treatment system. It briefly references the methods currently used to evaluate prediction accuracy and uncertainty and discusses the relevance of uncertainty evaluations in model applications. The paper aims to raise awareness and initiate a comprehensive discussion among professionals on model prediction accuracy and uncertainty issues. It also aims to identify future research needs. Ultimately the goal of such a discussion would be to generate transparent and objective methods of explicitly evaluating the reliability of model results, before they are implemented in an engineering decision-making context.

Key words | mathematical modelling, prediction accuracy, uncertainty, wastewater

E. Belia

Primodal Inc., 145 Aberdeen, Québec, QC G1R 2C9, Canada E-mail: belia@primodal.com

$Y$. Amerlinck

MOSTforWATER, Sint-Sebastiaanslaan 3a,

B-8500, Kortrijk, Belgium

E-mail: ya@mostforwater.com

L. Benedetti

BIOMATH, Ghent University,

Coupure links 653, B-9000, Ghent, Belgium

E-mail: Iorenzo.benedetti@ugent.be

B. Johnson

CH2M-Hill, 9193 South Jamaica Street,

Englewood, Denver, CO 80112, USA

E-mail: Bruce.Johnson2@ch2m.com

G. Sin K. V. Gernaey

Department of Chemical and Biochemical

Engineering, Technical University of Denmark DK-2800 Kgs, Lyngby, Denmark

E-mail: gsi@kt.dtu.dk; kvg@kt.dtu.dk

P. A. Vanrolleghem L. Rieger Département de génie civil, modelEAU, Université Laval,

Québec, QC G1K 7P4, Canada

E-mail: Peter.Vanrolleghem@gci.ulaval.ca; leiv.rieger@gci.ulaval.ca Kris.Villez@gmail.com

S. Gillot

Cemagref, UR HBAN, Parc de Tourvoie, BP 44

F-92163, Antony, France

E-mail: sylvie.gillot@cemagref.fr

Eawag, Swiss Federal Institute of Aquatic Science and Technology,

$\mathrm{CH}-8600$, Dübendorf, Switzerland

E-mail: Marc.Neumann@eawag.ch

A. Shaw

Black \& Veatch, 8400 Ward Parkway,

Kansas City, MO 64114, USA

E-mail: ShawAR@bv.com

K. Villez

Laboratory of Intelligent Process Systems (LIPS),

Purdue University, 480 Stadium Mall Drive,

West Lafayette, IN 47906, USA 


\section{INTRODUCTION}

Over the past 40 years, there has been a tremendous increase in the amount of knowledge the engineering and scientific communities have acquired in the field of wastewater treatment. This increased understanding has led us to shift our design approaches from using "rules of thumb" like $\mathrm{F} / \mathrm{M}$ ratios and $\mathrm{BOD}$ loading rates, to more accurate methods such as minimum sludge age and detailed influent characterisation. This increased knowledge has also resulted in our ability to construct mathematical models that describe the main processes that take place in wastewater treatment. In turn, the implementation of these models to engineering projects and the drive for their validation has deepened our understanding of the same processes. By applying these new tools we have improved our designs, made our plants more efficient and been able to comply with increasingly stringent regulations.

In spite of the advances we have made to date, our approach to plant design and optimisation still implies that we work in a well defined field (Gujer 2006). For example, we select one or two 'typical' flow and load scenarios, assumed to capture the conditions a plant will experience and size the plant to meet an average effluent standard. To account for the unpredictability of the influent wastewater and the much larger variability that the plant encounters, we are forced to incorporate safety factors in our design and build redundant systems on site (US EPA I993; WERF $2003 b$ ). These semi-arbitrary safety factors are lumped expressions of the individual sources of uncertainty underlying any treatment process. This lumping of uncertainty often results in overly conservative solutions. Most of the existing design guidelines do not incorporate explicit and objective methods for the evaluation of uncertainty. As a result, the risk associated with any engineering decision during a design, upgrade or optimisation project is accounted for implicitly through a combination of adhering to local or international guidelines, rules of thumb and the experience of the design engineer.

In the current regulatory environment of extremely low effluent nutrient standards (e.g. Chesapeake Bay area) and increased demands for operational efficiency, a new approach is required that provides us with an understanding of the main sources of uncertainty associated with each process (Gujer 2006). Moving away from lumped uncertainty safety factors should help us maximise existing plant capacity and avoid over-sizing new plants. A new approach should provide us with an objective way of discussing and evaluating risk and must allow the stakeholders involved in a particular project to discuss risk and who will assume it, openly.

Models can greatly assist us in the development of an objective, peer accredited methodology for evaluating process design and compliance risk. The way the models are formulated provides us with a structure which allows the identification and evaluation of the sources of uncertainty. They can thus provide the framework for the inclusion of uncertainty evaluations in plant design, upgrade and optimisation projects.

This paper summarizes the presentations and discussions held during the WWTmod2008 workshop on 'Model accuracy: dealing with uncertainties' (Belia et al. 2008). The workshop was organised in response to the increasing need of the engineering community to discuss uncertainty in model-based design and optimisation projects. This discussion was focused on identifying answering the question: how can we use the current modelling tools together with uncertainty analysis to develop a methodology that results in more efficient designs and explicit risk assessments?

The main objective of this paper is to serve as a problem statement of the issues surrounding uncertainty evaluations in wastewater treatment projects. The paper proposes a list of items that need to be covered in any work that aims to incorporate uncertainty evaluations into engineering projects. It also proposes an intuitive structure that allows clear identification of the sources of uncertainty introduced during a typical modelling project.

\section{CURRENT RESEARCH}

The incorporation of uncertainty evaluations in wastewater engineering is far less advanced compared to other fields. The academic and engineering communities have identified the need in our field and have tried to address it by 
proposing methods for the quantification of model prediction accuracy and uncertainty introduced during model development and application. Most publications to date deal with only a few of the sources of uncertainty in modelbased projects such as: wastewater influent and biokinetic parameters (Bixio et al. 2002; Melcer et al. 2003; Sin et al. 2009); model structure (Neumann \& Gujer 2008); sensor and measurement accuracy (Rieger et al. 2005) or prediction of future loads (Dominguez \& Gujer 2006; McCormick et al. 2007). There are also several publications on the topic of model prediction accuracy or goodness-of-fit evaluations for model calibration (Ahnert et al. 2007), uncertainty propagation (Benedetti et al. 2006) and incorporation of uncertainty for specific design objectives (Bixio et al. 2002; WERF 2003a; Neumann 2007). What is still lacking is a broad, comprehensive discussion of the sources of uncertainty and the evaluation methods applicable to wastewater treatment projects.

The challenges highlighted in the introduction and the needs identified in the brief literature review, point to the need for the development of a comprehensive protocol that incorporates uncertainty evaluations in model-based wastewater projects. The goal of such a protocol would be to generate transparent and objective methods of evaluating the reliability of model results. The protocol must be easily applicable and be scope-specific i.e. linked to the model objective.

\section{QUESTIONS THAT NEED TO BE ADDRESSED}

A comprehensive discussion on the subject of uncertainty and prediction accuracy must address several questions, including:

1. What are the concepts and definitions that need to be discussed so that a common language is established?

2. What are the sources of uncertainty?

3. What are the available methods, quantitative or qualitative, that can be used to evaluate model prediction accuracy and uncertainty?

4. How much effort should be put into the assessment of uncertainty and model prediction accuracy? Do all model applications require the same degree of detail of uncertainty evaluations?
- How much effort does one need to spend on data collection and reconciliation?

- What is the appropriate level of calibration/validation for a given task? Are dynamic solutions inherently more accurate than steady state solutions?

5. What confidence levels are required for different modelling objectives? How do we quantify risk?

6. What is the added benefit of including uncertainty evaluations in modelling projects?

- Could it provide a more objective way to determine appropriate safety factors?

- Could it generate specific data requirements depending on project objectives?

- Could it provide calibration guidelines for each model application?

7. How can uncertainty evaluations be incorporated into design?

8. How do stakeholders (technical and non-technical) communicate on the subject of uncertainty and risk?

It is beyond the scope of this paper to give answers to all of the questions listed above. The following sections summarize the presentations and discussions held during the WWTmod2008 workshop on 'Model accuracy: dealing with uncertainties'.

\section{TERMINOLOGY AND DEFINITIONS}

The first step in establishing an applicable methodology or protocol is to reach an understanding and agreement on terminology. That is, what do we mean when using such terms as uncertainty, accuracy, precision, confidence, error and reliability? These terms have established definitions in specific fields, e.g. in data quality management or chemical analysis. However, when we expand their definition to cover model quality, even for the most widely used terms there appears to be a lack of consensus. For the benefit of the readers, a selection of key terms and definitions has been included in this paper. The list of selected definitions has been compiled by the authors from different sources (Taylor \& Kuyatt 1994; Carstensen et al. I997; Dochain \& Vanrolleghem 200I; ISO 15839 2003). 


\section{Trueness of measurement}

The degree of closeness of the expected value of a measurement or estimate to an accepted reference value. Expected values are obtained by averaging over repeated measurements or estimates. Trueness is an expression of systematic error.

\section{Precision of measurement}

The degree of similarity or closeness between repeated measurements or estimates of the same variable, subjected to the same sources of uncertainty. Precision is an expression of random error and does not relate to the true or specified value.

\section{Confidence interval}

Instead of estimating the parameter by a single value, an interval or range likely to include the parameter is given. How likely the interval is to contain the parameter is determined by the confidence level.

\section{Model prediction accuracy}

An estimate of how close a model predicted quantity is to its measured value. The difference between model predictions and the corresponding measured values of a calibration or validation data set, during a model run.

\section{Model calibration}

The (mostly iterative) adjustment of any model parameter (physical, operational, kinetic, stoichiometric, settling,...) to improve the fit to measured data.

\section{Model validation}

The comparison of the predictions of a calibrated model to a different and independent data set not used for calibration.

\section{Uncertainty}

The degree of lack of knowledge about a system or process or degree of inability to exactly describe its existing state and/or behaviour.
Uncertainty can be further classified by its nature and level as detailed below (Walker et al. 2003; Refsgaard et al. 2007).

\section{Nature of uncertainty}

Reducible-Uncertainty that can be reduced with further research/efforts. (e.g. experimental determination of kinetic parameters).

Irreducible-Uncertainty due to the inherent variability of a system that cannot be reduced with any further research/efforts (e.g. rainfall, toxic spills).

\section{Level of uncertainty:}

Quantifiable uncertainty can be quantified and described in a statistical sense and can be attributed to uncertainties surrounding measurement and sampling errors, probabilities, etc.

Scenario uncertainty can be described with qualitative estimations of possible outcomes that may develop in the future. Realistic assumptions about relationships and/or driving forces within the model can be established. It is not possible, however, to derive the probabilities of the scenarios taking place.

Recognized ignorance is the state where fundamental uncertainty is acknowledged to exist and the scientific basis is insufficient to develop functional relationships, statistics, or scenarios.

Total ignorance is defined as the state where a deep level of uncertainty exists. It is unknown what is unknown.

Figure 1 shows a schematic representation of the levels of uncertainty ranging from full knowledge of all outcomes (determinism) to a complete lack of knowledge (indeterminacy).

\section{SOURCES OF UNCERTAINTY}

To date most researchers have classified the sources of uncertainty from the perspective of where they are located in a generic model (Walker et al. 2003; Refsgaard 


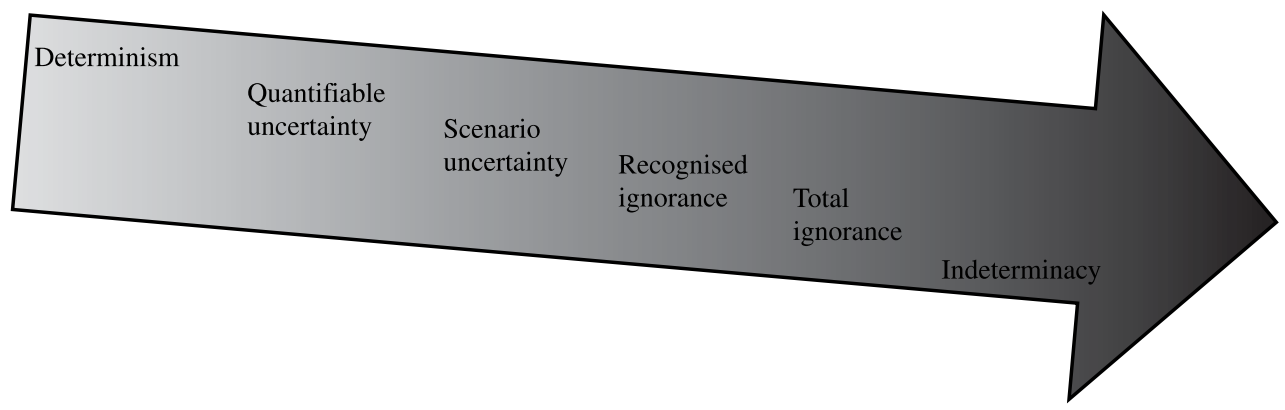

Figure 1 | Levels of uncertainty.

et al. 2004). Thus, they identify three or four main areas that introduce uncertainties to model predictions: model inputs, i.e. any type of data needed to perform a simulation (e.g. influent flow, wastewater characteristics), model structure (e.g. activated sludge model, clarifier model) and model parameters. Uncertainty in the inputs is due to random variations of the system (e.g. weather) and to errors in the measurements (e.g. imprecise sampling and measurement techniques). Uncertainty in the model is due to our incomplete understanding of the modelled processes and/or the simplified descriptions of the processes we chose to include in our models. A fourth source of uncertainty results from the implementation of the models in software packages (e.g. numerical integration, bugs, solver settings) (Yuan et al. I997; Copp 2002; Reichert 2006). Table 1 shows the classification of the sources of uncertainty based on the location of uncertainty as mentioned above.

To provide a more intuitive method of identifying the sources of uncertainty, it is proposed that the focus be shifted from the location of uncertainty within the model to when this uncertainty is introduced during a typical modelling project. To aid this analysis, the typical steps of a standard modelling project can be used (Langergraber et al. 2004; Refsgaard et al. 2005; Sin et al. 2005; IWA GMP-TG 2008). The five steps, shown in the first row of Figure 2 are an intuitive sequence of tasks as suggested by the IWA Task Group on Good Modelling Practice Guidelines for use of activated sludge models (IWA GMP-TG 2008).

Uncertainty can be identified and evaluated at key times during a project as suggested in the Harmoni-CA report (Refsgaard et al. 2004) and shown in Figure 2. Figure 2 also includes a list of items, for each project step, which need to be selected or decided upon and which identify a location of uncertainty. The figure therefore combines the traditional location of uncertainty within the model with a project-step oriented or sequential approach.

Table 2 includes the sources of uncertainty introduced during a typical modelling project. It provides brief explanations for each of the sources of uncertainty and classifies them according to the nature of uncertainty as reducible or irreducible. In addition, a proposed characterisation of uncertainty on the basis of its level has been included. Table 2 shows that each of the building blocks of a plant model (influent, activated sludge model, final clarifier model, etc) as implemented in a simulator introduces uncertainties that can be identified and evaluated.

\section{QUANTITATIVE EVALUATION METHODS}

As can be seen from Table 2, a significant number of the sources of uncertainty associated with a wastewater modelling project are quantifiable. Several methods exist in the literature for the evaluation of uncertainty as well as model prediction accuracy. Establishing objective methods for the quantification of the prediction accuracy of a model is very important if models are to be used as the framework around which a protocol for uncertainty evaluations is to be structured. The methods presented below are examples of the most frequently used ways of quantifying model prediction accuracy and uncertainty. The list needs to be expanded to cover both quantitative and qualitative methods that will allow the assessment of all the sources 
Table 1 | Location of uncertainty

\begin{tabular}{|c|c|c|}
\hline Area & Details & Examples \\
\hline \multirow[t]{6}{*}{ Inputs } & Influent data & Current and future predicted flow, COD, ammonia \\
\hline & Physical data & Tank volume and geometry \\
\hline & Operational settings & DO set points \\
\hline & Performance data & Effluent data, reactor concentrations \\
\hline & Additional info & Input from connected systems e.g. sewers, catchment \\
\hline & $\ldots$ & $\ldots$ \\
\hline \multirow[t]{3}{*}{ Model } & Model structure & $\begin{array}{l}\text { Influent model, hydraulic model, aeration system model, } \\
\text { process models (biological, settling,...) }\end{array}$ \\
\hline & Interfaces between models & $\begin{array}{l}\text { Waste activated sludge pumped to an anaerobic digester; digester } \\
\text { effluent pumped to sludge treatment }\end{array}$ \\
\hline & $\cdots$ & $\cdots$ \\
\hline \multirow[t]{4}{*}{ Model parameters } & Hydraulic & Number of tanks in series \\
\hline & Biokinetic & Maximum growth rate \\
\hline & Settling & Settling coefficients \\
\hline & $\ldots$ & $\ldots$ \\
\hline \multirow[t]{5}{*}{ Software (model technical aspects) } & Solver settings & \\
\hline & Numerical approximations & \\
\hline & Software limitations & \\
\hline & Bugs & \\
\hline & $\ldots$ & $\ldots$ \\
\hline
\end{tabular}

of uncertainty deemed important for a particular project. Systematic procedures, together with their practical application, should also be elaborated and proposed.

\section{Evaluating model prediction accuracy}

In cases where plant data are available, evaluations of predictive quality of the model start with an assessment of the model prediction error. This includes qualitative (visual, graphical) and quantitative (statistical) comparisons of model results with field observations. Most often the only form of evaluation undertaken is visual comparison of time series plots of the observations with the model predictions. The simplest quantitative analysis is the generation of a scatter plot of observations vs. model predictions or plots of the residuals (measured value minus predicted value). A more involved analysis may include the use of descriptive statistics and more advanced goodness-of-fit measures such as correlation coefficients. Examples of the statistical coefficients typically used are shown in Table 3 (Ramaswari et al. 2005; Ahnert et al. 2007 and Sin et al. 2008).
Typically, these coefficients will be calculated for the model predictions that are the most important indicators of plant performance for the specific modelling objective (ammonia, nitrates, phosphates, MLSS, etc.). In this way, an objective measure can be used to support subjective evaluation methods such as visual inspection of data fits. The use of statistical measures assists the comparison of the prediction accuracy obtained in different modelling projects.

Currently there does not appear to be a consensus on how to apply these statistical coefficients quantifying model prediction accuracy to wastewater models nor has there been an evaluation of their applicability at different value ranges. An evaluation of the advantages and disadvantages of each method along with suggested application guidelines is required.

Although statistical comparisons provide an objective, reproducible method for evaluating how well model predictions fit measured data, caution should be exercised when making judgements on how good a particular model is based on statistical calculations. Statistical evaluations provide very little insight into why model predictions 


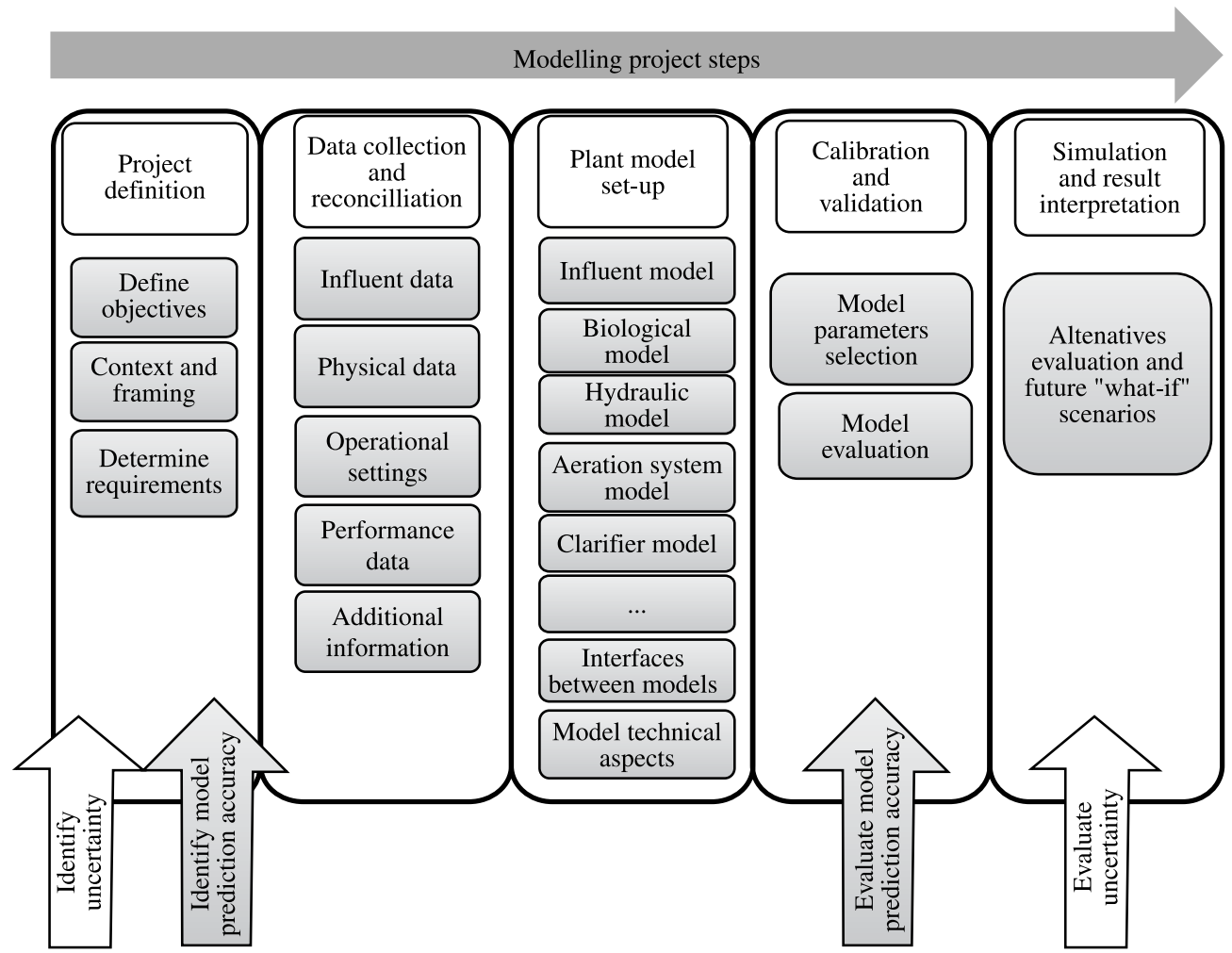

Figure 2 Typical modelling project steps including the instances where model uncertainty and prediction accuracy should be identified and evaluated (adapted from Refsgaard et al. 2004 and IWA GMP-TG 2008).

deviate from measured data and should be combined with other assessments that are based on process expertise.

\section{Evaluating uncertainty}

As shown in Table 2, evaluating uncertainties for any mathematical model begins by specifying the scope of the model, which includes identifying the relevant sources of uncertainty. Following this, each of the uncertainties that are considered significant to the specific model application can be evaluated and where possible quantified. In the literature one finds several methods that can be used for the quantification of uncertainty, a number of which have been listed below.

1. Methods used to characterize and prioritize uncertainty:

- data quality evaluations

- expert elicitation

- parameter estimation

- sensitivity analysis
- techniques developed for specific applications (the Numerical, Unit, Spread, Assessment and Pedigree (NUSAP) method, the uncertainty matrix)

2. Methods aiming to increase the quality of information:

- quality assurance

- extended peer review

- stakeholder involvement

3. Methods used to quantify and propagate uncertainty in model calculations to evaluate uncertainty in model outcome:

- Gaussian error propagation

- Monte Carlo simulation

- Inverse modelling (multiple model simulation)

- Scenario analysis

A detailed discussion of these methods is beyond the scope of this paper. However, it is important to highlight 
Data collection and reconcilliation

\section{Details of each step}

Project definition Objectives

Context and framing

Requirements

Influent data

Physical data

Operational settings

Performance data

Additional information Equipment failures what type of data flow splits pumped flows
Nature and source of uncertainty

Level of uncertaint

The required prediction accuracy of the

model is decided at this stage of the project.

This will define which of the uncertainty

items listed below will be taken into accoun

daries of the system to be modelled. Biological treatment only, whole plant or sewer and river

Level of model prediction accuracy,

Flow rate, concentrations, influent characterisation data, data from other models and other systems like sewers

Process flow diagram, active (effective) tank volumes, clarifier surface areas,

Controller set-points, valve positions,

Effluent data and reactor concentrations such as MLSS (when not used as controller set-points) the real system like weather, unexpected

Quantifiable, cenario, recognised demographic changes, unexpected factory ignorance hutdowns

Reducible: due to data collection e.g. sampling method, location, frequency, accuracy of sensors, accuracy of analytical techniques

Irreducible: due to the unpredictable and dynamic behaviour of structures like splitters to flow changes

Reducible: due to e.g. unknown true volume constructed or operational depth of

structures

Irreducible: due to the unpredictability of operator decisions

Reducible: due to actions different from planned or changes not logged, e.g. a chang in set-points, incorrect controller set up e.g. scales different between field and

control room

Irreducible: due to the inherent variability of the real system e.g. response of microbia consortium

Reducible: due to data collection issues

Irreducible: e.g. due to unexpected

equipment failures

\section{Quantifiable}

Scenario

Quantifiable

Quantifiable, scenario

Recognised ignorance, quantifiable

Quantifiable, scenario, recognised ignorance

Quantifiable

Quantifiable, scenario, recognised ignorance 
Table 2 | (continued)

Influent dynamics, characteristics, influent fractions

Biological mode

Model structure: processes (conversion, separation), calculation of composite variables, type of mathematical expression used to describe processes (Monod vs. enzymatic kinetics)

Hydraulic model calibrated, time varying

Model structure: transport and mixing processes, number of trains, number of tanks in series

Model parameters: fixed, a priori chosen, calibrated, time varying

Aeration system model Model structure: gas transfer processes, mechanical system details

Model parameters: fixed, a priori chosen, calibrated, time varying

Clarifier mode
Model structure: separation processes, calculation of composite variables and type of mathematical expression used to describe processes (1-D, 2-D, CFD analysis)

Model parameters: fixed, a priori chosen, calibrated, time varying
Reducible: due to simplifications of influent dynamics (applying a generic diurnal pattern to average vs. constructing a dynamic profile of the whole sewer system), due to simplifications of influent characteristics (fixed ratios for influent fractions)

Irreducible: due to the inherent variability of Recognised ignorance the real system

Reducible: due to simplifications in model structure e.g. processes not included, processes included in simplified form (one step vs. two step nitrification), due to the choice of mathematical description of processes

Reducible: due to our lack of knowledge of the appropriate value

Reducible: due to the simplification of transport and mixing processes in models, nadequate spatial resolution (CSTRs vs.

CFD, selection of number of trains to model, number of tanks in series)

Reducible: due to the simplification of gas transfer processes and aeration system

Reducible: due to simplifications in model structure e.g. processes not included,

processes included in simplified form as well as due to the choice of mathematical description of processes

Irreducible: due to inherently varying biomass settling properties

Reducible: due to our lack of knowledge of the appropriate value
Quantifiable, scenario

\section{Quantifiable}

Quantifiable, scenario

Quantifiable, scenario

Quantifiable, scenario

Quantifiable, scenario 
Table 2 | (continued)

\begin{tabular}{|c|c|c|c|c|c|}
\hline \multicolumn{3}{|c|}{ Typical modelling project steps } & \multirow{2}{*}{$\begin{array}{l}\text { Details of each step } \\
\text { Control loops, sensors, actuators, time } \\
\text { variation of set-points }\end{array}$} & \multirow{2}{*}{$\begin{array}{l}\text { Nature and source of uncertainty } \\
\text { Reducible: due to the oscillation of the } \\
\text { aeration system, time delays in control loops, } \\
\text { non-linearity of actuators }\end{array}$} & \multirow{2}{*}{$\begin{array}{l}\text { Level of uncertainty } \\
\text { Quantifiable, scenario }\end{array}$} \\
\hline & & $\begin{array}{l}\text { Controllers in plant } \\
\text { operations }\end{array}$ & & & \\
\hline & & $\begin{array}{l}\text { Interfaces between } \\
\text { models }\end{array}$ & $\begin{array}{l}\text { Use of one or several sets of state } \\
\text { variables, calculation of composite } \\
\text { variables }\end{array}$ & $\begin{array}{l}\text { Reducible: due to the aggregation of state } \\
\text { variables }\end{array}$ & Quantifiable \\
\hline & & $\begin{array}{l}\text { Model technical } \\
\text { aspects }\end{array}$ & $\begin{array}{l}\text { Numerics: solver selections \& } \\
\text { settings, bugs }\end{array}$ & $\begin{array}{l}\text { Reducible: due to numerical approximations } \\
\text { and software bugs }\end{array}$ & $\begin{array}{l}\text { Quantifiable, } \\
\text { recognised ignorance }\end{array}$ \\
\hline & & & $\begin{array}{l}\text { Simulators: limitations of simulation } \\
\text { platforms }\end{array}$ & & \\
\hline \multirow{2}{*}{\multicolumn{2}{|c|}{$\begin{array}{l}\text { Calibration } \\
\text { Validation }\end{array}$}} & $\begin{array}{l}\text { Model parameter } \\
\text { selection }\end{array}$ & $\begin{array}{l}\text { Selection of model parameters for e.g. } \\
\text { biological, separation models that need to } \\
\text { be adjusted }\end{array}$ & $\begin{array}{l}\text { Model prediction error calculations. } \\
\text { Uncertainty analysis of calibration \& } \\
\text { validation parameters }\end{array}$ & N/A \\
\hline & & Model evaluation & $\begin{array}{l}\text { Assessment of model prediction error for } \\
\text { calibration \& validation data sets through } \\
\text { the implementation of quantification } \\
\text { methods such as statistical coefficients }\end{array}$ & & N/A \\
\hline Simulation & & $\begin{array}{l}\text { Alternatives } \\
\text { evaluation, future } \\
\text { "what-if" scenarios }\end{array}$ & $\begin{array}{l}\text { Generation of model desired results } \\
\text { (probability distributions, statistics) }\end{array}$ & $\begin{array}{l}\text { Post-calibration uncertainty analysis of } \\
\text { simulations (sensitivity and Monte Carlo } \\
\text { uncertainty analysis) }\end{array}$ & N/A \\
\hline
\end{tabular}


Table 3 | Statistical coefficients used to quantify model prediction accuracy

\begin{tabular}{ll} 
Coefficient & Definition/formula \\
\hline Residuals & $r_{i}=C_{\mathrm{pr}}^{i}-C_{\mathrm{ob}}^{i}$ \\
Mean of residuals (Bias) & $m=\frac{1}{n} \sum_{i=1}^{n} r_{i}$ \\
Root-mean-square of residuals (RMSR) & $\sqrt{\frac{1}{n} \sum_{i=1}^{n}\left(r_{i}\right)^{2}}$ \\
Coefficient of determination & $R^{2}=1-\frac{\sum_{i=1}^{n}\left(C_{\mathrm{ob}}^{i}-C_{\mathrm{pr}}^{i}\right)^{2}}{\sum_{i=1}^{n}\left(C_{\mathrm{ob}}^{i}-\mu\right)^{2}}$ \\
\hline
\end{tabular}

Where: $\mathrm{N}=$ Number of prediction/observation pairs, $C_{\mathrm{pr}}^{i}=$ model prediction at time instant $i, C_{\mathrm{ob}}^{i}=$ observation (measurement) at time $i, m=$ mean of residuals, $\mu=$ mean of observations.

that they are available and widely used in other fields. During the development of the proposed protocol for the inclusion of uncertainty evaluations in engineering projects, these methods need to be assessed for their applicability in the wastewater field. Further details and examples of the implementation of the methods listed above can be found in WERF (2003a), Refsgaard et al. (2005), Benedetti et al. (2006) and Ahnert et al. (2007) among others.

\section{THE RELEVANCE OF MODEL SCOPE AND APPLICATION IN THE EVALUATION OF UNCERTAINTY}

Models can be used in multiple stages of a treatment plant's life cycle: research, pre-design, detailed design, start up, process optimization, plant performance analysis, process control, plant upgrades, forecasting, education and training (students and operators) and operator decision support. These different applications require different levels of confidence in model predictions and hence different levels of effort spent on data collection, model calibration and uncertainty evaluations. For example, a preliminary design project requires results quickly and therefore steady state simulations and a series of sensitivity analyses of the parameters where the larger amount of uncertainty lies would suffice. A model used for operational decision support will require fast, robust and highly predictive results. In this case a dynamic calibration and detailed uncertainty analysis may be required.

Because the monetary cost and time input increases with increased requirements, it is important that the engineering community reaches a consensus on the level of confidence and effort required to achieve each project goal. This will be dictated by the intended model application (IWA GMP-TG 2008). One can surmise that increased effort is required with increasing complexity of the modelling objective. The development of a protocol that incorporates uncertainty evaluations will provide quantitative measures to the qualitative criteria discussed above.

\section{CONCLUSIONS}

As regulatory demands require plant owners to design and operate processes close to their limits while at the same time increasing energy efficiency and reducing greenhouse gas emissions, identifying and quantifying the uncertainties involved in a new design or plant upgrade becomes crucial. This is especially evident during final bid selection processes, when plant owners have to select a design from a list of proposals that involve different engineering approaches, different processes and costs, all meeting the same objective. One of the parameters that differentiate the bids, not explicitly stated in the proposals, is the uncertainty in each design and the risk that the engineers have assumed in each of the proposed solutions.

Historically the uncertainty involved in predicting the performance of wastewater treatment plants has been addressed through the incorporation of safety factors which are essentially lumped expressions of all the sources of uncertainty. The implementation of models provides practitioners with a structure which allows the systematic identification and quantification of the majority of the sources of uncertainty. It allows them to steer away from semi-arbitrary safety factors, which often result in overly conservative solutions. Instead, safety factors can be derived from quantifications of the uncertainty in each model. This new approach will provide stakeholders with the ability to explicitly quantify uncertainties and include risk evaluations in their decision making process. It should generate new ways of assessing process performance such as confidence intervals or probability curves which will lead to the estimation of accurate design factors.

To initiate the discussion of uncertainty evaluation in the wider engineering community, the work of academics in the field of wastewater and elsewhere needs to be combined with the needs of the engineers implementing modelling for 
various applications. To this end the authors of this paper are proposing a number of items that need to be discussed. These range from reaching an agreement on terminology to identifying the sources of uncertainty and the available methods for their evaluation. The authors also note the need for the development of a protocol that helps engineers to include uncertainty evaluations in model-based design and optimisation projects. Leveraging the power of models can facilitate the difficult task of evaluating risk.

\section{$\overline{\text { OUTLOOK }}$}

This paper outlines the issues surrounding uncertainty in wastewater treatment and identifies the need for the development of a protocol that incorporates uncertainty evaluations in modelling projects. The goal of such a protocol would be to generate transparent, peer accredited methods of evaluating the reliability of model results. The same methods could be used i) for model-based plant design procedures, ii) to generate design factors and iii) define data requirements. As a first step, the following tasks need to be undertaken:

1. Establish the state of the art in the field of uncertainty evaluation for wastewater treatment projects

2. Review the current practice in respect to assessing risk in design, upgrade or optimisation projects

The work undertaken as part of the above tasks will provide the engineering community with the necessary information to be able to:

- Propose a set of terms and definitions and decide on a common terminology.

- Propose a comprehensive list of the sources of uncertainty.

- Document and evaluate the existing methods for assessing uncertainty.

- Identify gaps in current knowledge and define the developments required to provide adequate tools for practitioners to implement uncertainty evaluations in projects.

- Incorporate knowledge on uncertainty evaluation from other disciplines.

This will enable to complete follow-up tasks necessary for the development of a protocol. These may include:
1. The development of new or the modification of existing uncertainty assessment and evaluation methods.

2. The generation of transparent, peer accredited methods to replace current safety factors with new design factors, calibration requirements, data requirements, etc.

3. The development of a communication framework on uncertainty to address the "non-expert" community including regulators.

These are ambitious and demanding goals which have important implications to the current practice of wastewater treatment profession and industry. Any undertaking to achieve these goals requires a multi-disciplinary collaboration and multi-stakeholder involvement ranging from academia and consultants to regulators and professional associations.

\section{ACKNOWLEDGEMENTS}

The authors would like to acknowledge an extended group of reviewers that have contributed to the work presented in this paper, namely: Jens Alex (ifak, Germany), Eduardo Ayesa (CEIT, Spain), Joe Husband (Malcolm Pirnie, USA), Guenter Langergraber (BOKU, Austria), Paul Lessard (Université Laval, Canada), Jeff McCormick (Carollo Engineers, USA), Maureen O' Shaughnessy (ASA, USA), Mark van Loosdrecht (TU Delft, The Netherlands), Stefan Weijers (Waterschap De Dommel, The Netherlands). The authors would also like to thank all the WWTMod2008 workshop participants for their contribution.

\section{REFERENCES}

Ahnert, M., Blumensaat, F., Langergraber, G., Alex, J., Woerner, D., Frehmann, T., Halft, N., Hobus, I., Plattes, M., Spering, V. \& Winkler, S. 2007 Goodness-of-fit measures for numerical modelling in urban water management-a summary to support practical applications. In: Proceedings 10th LWWTP Conference, 9-13 September 2007, Vienna, Austria.

Belia, E., Amerlinck, Y., Benedetti, L., Johnson, B., Sin, G. \& Vanrolleghem, P. A. 2008 WWTmod2008 Workshop. Modelling accuracy: dealing with uncertainties. In: Proceedings 1st IWA/WEF Wastewater Treatment Modelling Seminar, Mont-Sainte-Anne, Québec, Canada, June 1-3, 2008. 
Benedetti, L., Bixio, D. \& Vanrolleghem, P. A. 2006

Benchmarking of WWTP design by assessing costs, effluent quality and process variability. Water Sci. Technol. 54(10), 95-102.

Bixio, D., Parmentier, G., Rousseau, D., Verdonck, F., Meirlaen, J., Vanrolleghem, P. A. \& Thoeye, C. 2002 A quantitative risk analysis tool for design/simulation of wastewater treatment plants. Water Sci. Technol. 46(4-5), 301-307.

Carstensen, J., Vanrolleghem, P. A., Rauch, W. \& Reichert, P. 1997 Terminology and methodology in modelling for water quality management-a review. Water Sci. Technol. 36(5), 157-168.

Copp, J. B. (ed.) 2002 The COST Simulation BenchmarkDescription and Simulator Manual. ISBN 92-894-1658-0, Office for Official Publications of the European Communities, Luxembourg.

Dochain, D. \& Vanrolleghem, P. A. 20or Dynamical Modelling and Estimation in Wastewater Treatment Processes. IWA Publishing, London, UK.

Dominguez, D. \& Gujer, W. 2006 Evolution of a wastewater treatment plant challenges traditional design concepts. Water Res. 40(7), 1389-1396.

Gujer, W. 2006 Activated sludge modelling: past, present and future. Water Sci. Technol. 53(3), 111-119.

ISO 158392003 Water Quality-On-Line Sensors/Analysing Equipment for Water-Specifications and Performance Tests, Geneva, Switzerland.

IWA Task Group on Good Modelling Practice-Guidelines for use of activated sludge models 2008 Website: www.modeleau.org/ GMP_TG

Langergraber, G., Rieger, L., Winkler, S., Alex, J., Wiese, J., Owerdieck, C., Ahnert, M., Simon, J. \& Maurer, M. 2004 A guideline for simulation studies of wastewater treatment plants. Water Sci. Technol. 50(7), 131-138.

McCormick, J. F., Johnson, B. \& Turner, A. 2007 Analyzing risk in wastewater process design: using Monte Carlo simulation to move beyond conventional design methods. In: Proceedings of the 80th Annual Water Environment Federation Technical Exhibition and Conference, San Diego, California, USA, October 13-17, 2007.

Melcer, H., Dold, P. L., Jones, R. M., Bye, C. M., Takacs, I., Stensel, H. D., Wilson, A. W., Sun, P. \& Bury, S. 2003 Methods for Wastewater Characterisation in Activated Sludge Modelling. Water Environment Research Foundation (WERF), Alexandria, VA, USA.

Neumann, M. B. 2007 Uncertainty analysis for performance evaluation and design of urban water infrastructure. $\mathrm{PhD}$ Thesis, ETH Zurich, Switzerland.

Neumann, M. B. \& Gujer, W. 2008 Underestimation of uncertainty in statistical regression of environmental models: influence of model structure uncertainty. Environ. Sci. Technol. 42(11), 4037-4043.

Ramaswari, A., Milford, J. B. \& Small, M. J. 2005 Integrated Environmental Modeling. Pollutant Transport, Fate and Risk in the Environment. John Wiley \& Sons, Inc. ISBN: 978-0-471-35953-1.
Refsgaard, J. C., van der Sluijs, J. P., Højberg, A. L. \& Vanrolleghem, P. A. 2004 Harmoni-CA Guidance 1: Uncertainty Analysis. A research project supported by the European Commission under the 5th Framework Programme and contributing to the implementation of the Key Action "Sustainable Management and Quality of Water" within the Energy, Environment and Sustainable Development. Contract No: EVK1-2001-00192.

Refsgaard, J. C., Henriksen, H. J., Harrar, W. G., Scholten, H. \& Kassahun, A. 2005 Quality assurance in model based water management-review of existing practise and outline of new approaches. Environ. Modell. Softw. 20, $1201-1215$.

Refsgaard, J. C., van der Sluijs, J. P., Højberg, A. L. \& Vanrolleghem, P. A. 2007 Uncertainty in the environmental modelling process-a framework and guidance. Environ. Modell. Softw. 22, 1543-1556.

Reichert, P. 2006 A standard interface between simulation programs and systems analysis software. Water Sci. Technol. 53(1), 267-275.

Rieger, L., Thomann, M., Gujer, W. \& Siegrist, H. 2005 Quantifying the uncertainty of on-line sensors at WWTPs during field operation. Water Res. 39(20), 5162-5174.

Sin, G., Van Hulle, S. W. H., De Pauw, D. J. W., van Griensven, A. \& Vanrolleghem, P. A. 2005 A critical comparison of systematic calibration protocols for activated sludge models: a SWOT analysis. Water Res. 39, 2459-2474.

Sin, G., De Pauw, D. J. W., Weijers, S. \& Vanrolleghem, P. A. 2008 An efficient approach to automate the manual trial and error calibration of activated sludge models. Biotechnol. Bioeng. 100, 516-528.

Sin, G., Gernaey, K. V., Neumann, M. B., van Loosdrecht, M. C. M. \& Gujer, W. 2009 Uncertainty analysis in WWTP model applications: a critical discussion using an example from design. Water Res. 43(11), 2894-2906. DOI: 10.1016/ j.watres.2009.03.048.

Taylor, B. N. \& Kuyatt, C. E. I994 NIST Technical Note 1297. Guidelines for Evaluating and Expressing the Uncertainty of NIST Measurement Results. Physics Laboratory, National Institute of Standards and Technology.

US EPA (1993) Manual: Nitrogen control. US EPA 625/R-93/010.

Walker, W. E., Harremoes, P., Rotmans, J., van der Sluijs, J. P., van Asselt, M. B. A., Janssen, P. \& Krayer von Krauss, M. P. 2003 Defining uncertainty: a conceptual basis for uncertainty management in model-based decision support. Integr. Assess. 4(1), 5-17.

WERF 2003a Tools for Rating the Capacity of Activated Sludge Plants. WERF Report: Treatment Processes/Systems (Project 00-CTS-3). ISBN: 9781843396789.

WERF 2003b Efficient Redundancy Practices. (Project 00-CTS-5). ISBN:1-893664-70-8.

Yuan, Z., Vanrolleghem, P. A. \& Vansteenkiste, G. 1997 Modeling error identification of activated sludge models. Water Sci. Technol. 36(5), 81-88. 